\title{
Development of a LAG-3 Immunohistochemistry Assay for Melanoma
}

Lori Johnson, BS, ${ }^{*}$ Bryan McCune, MD, ${ }^{*}$ Darren Locke, PhD, ${ }^{\dagger \ddagger}$ Cyrus Hedvat, MD, PhD, ${ }^{\ddagger}$ John B. Wojcik, MD, PhD, ${ }^{\dagger}$ Caitlin Schroyer, BS, ${ }^{\star}$ Jim Yan, PhD, ${ }^{*}$ Krystal Johnson, MD, ${ }^{*}$ Angela Sanders-Cliette, MD, ${ }^{*}$ Sujana Samala, MD, ${ }^{*}$ Lloye M. Dillon, $\mathrm{PhD},{ }^{\dagger}$ Steven Anderson, $\mathrm{PhD},{ }^{*}$ and Jeffrey Shuster, $\mathrm{PhD}{ }^{*}$

*Labcorp of America, Burlington, NC, USA; and ${ }^{\dagger}$ Bristol Myers Squibb, Princeton, NJ, USA. ${ }^{\ddagger}$ At the time the study was performed.

Corresponding author: Jeffrey Shuster, PhD, Companion Diagnostics, Labcorp Drug Development, 100 Perimeter Park, Suite C, Morrisville, NC 27560, USA. Email: jeffrey.shuster@labcorp.com. Phone: 919-388-5536

Current word count: $2921 / 3000$ words

Current figure/table count: 4 figures, 4 tables. 4 supplemental figures, 3 supplemental tables.

References: 32 


\section{Abstract (248/250)}

Aims: A robust immunohistochemistry $(\mathrm{IHC})$ assay was developed to detect lymphocyte-activation gene 3 (LAG-3) expression by immune cells (ICs) in tumor tissues. LAG-3 is an immuno-oncology target with demonstrable clinical benefit, and there is a need for a standardized, well-characterized assay to measure its expression. This study aims to describe LAG-3 scoring criteria and present the specificity, sensitivity, analytical precision, and reproducibility of this assay.

Methods: The specificity of the assay was investigated by antigen competition and with LAG3 knockout cell lines. A melanin pigment removal procedure was implemented to prevent melanin interference in IHC interpretation. Formalin-fixed, paraffin-embedded (FFPE) human melanoma samples with a range of LAG-3 expression levels were used to assess the sensitivity and analytical precision of the assay with $a \geq 1 \%$ cutoff to determine LAG-3-positivity. Interobserver and intraobserver reproducibility were evaluated with 60 samples in intralaboratory studies and 70 samples in interlaboratory studies.

Results: The LAG-3 IHC method demonstrated performance suitable for analysis of LAG-3 IC expression in clinical melanoma samples. The pretreatment step effectively removed melanin pigment that could interfere with interpretation. LAG-3 antigen competition and analysis of $L A G 3$ knockout cell lines indicated that the 17B4 antibody clone binds specifically to LAG-3. The intrarun repeatability, interday, interinstrument, interoperator, and interreagent lot reproducibility demonstrated a high scoring concordance (>95\%). The interobserver and intraobserver reproducibility and overall 
interlaboratory and intralaboratory reproducibility also showed high scoring concordance $(>90 \%)$.

Conclusions: We have demonstrated that the assay reliably assesses LAG-3

expression in FFPE human melanoma samples by IHC.

Key Words: immunohistochemistry, melanoma, molecular pathology 
Key messages ( $3-5$ sentences maximum)

What is already known on this topic: Lymphocyte-activation gene 3 (LAG-3) is an immune checkpoint receptor expressed on immune cells that limits T-cell activity and is being actively explored as a target for immunotherapy.

What this study adds: An immunohistochemistry assay was developed to detect the LAG-3 protein in formalin-fixed paraffin-embedded human tumor tissue specimens. This study describes scoring criteria and shows the specificity, sensitivity, analytical precision, and reproducibility of this assay as an aid to determine LAG-3 expression in melanoma patients using $a \geq 1 \%$ expression on immune cells threshold.

\section{How this study might affect research, practice or policy:}

The study describes a key immuno-oncology checkpoint immunohistochemistry assay that is robust and suitable for clinical trials. The assay was used in RELATIVITY-047 (NCT03470922), a phase 2/3 clinical trial that compared combined nivolumab and relatlimab treatment with nivolumab monotherapy, to stratify patients based on the percentage of LAG-3-positive immune cells within the tumor region. This assay is also being used in several ongoing clinical trials evaluating clinical response to relatlimab. 


\section{INTRODUCTION}

Immune checkpoint inhibitor-based therapies have greatly improved clinical outcomes across multiple disease settings,[1, 2] including advanced melanoma,[3-5] non-small cell lung cancer, $[6,7]$ squamous cell carcinoma of the head and neck, $[8,9]$ and urothelial carcinoma, $[10,11]$ among others. However, given the multiple mechanisms of immune evasion utilized by cancer cells, inhibition of a single immune checkpoint, such as programmed death-1 (PD-1), may not be sufficient to overcome immune suppression.[12, 13] Novel immuno-oncology (I-O) combinations, including dual checkpoint inhibition, may be necessary to enhance efficacy and to improve the durability of patient responses.

Lymphocyte-activation gene 3 (LAG-3, CD223) is a cell-surface molecule expressed on activated CD4+ and CD8+ T cells, as well as other immune cells (ICs) including regulatory T cells, natural killer cells, B cells, macrophages, and dendritic cells, and is under investigation as an I-O therapy target.[13-17] The interaction of LAG-3 with its ligands, the major histocompatibility complex II (MHCII), and fibrinogen-like protein 1 (FGL-1), recently discovered as a LAG-3 ligand, initiates an inhibitory signal.[13, 18, 19] This signal can impair T-cell function, activation, and proliferation, decrease production of and response to proinflammatory cytokines, and decrease the development of memory T cells.

Preclinical data indicate that simultaneous activation of the LAG-3 and PD-1 pathways in tumor-infiltrating lymphocytes results in greater T-cell exhaustion than either pathway alone, and dual inhibition of these pathways may improve T-cell function and increase immune response.[20] Furthermore, combined therapy with anti-LAG-3 and anti-PD-1 
agents in fibrosarcoma and colorectal adenocarcinoma mouse models resulted in synergistic antitumor activity.[16] The clinical efficacy of combining relatlimab, an antiLAG-3 antibody, with nivolumab, an anti-PD-1 agent, was previously demonstrated in patients with previously untreated metastatic or unresectable melanoma by the phase 2/3 RELATIVITY-047 clinical trial (NCT03470922).[21] RELATIVITY-047 demonstrated superior progression-free survival (PFS) for relatlimab combined with nivolumab versus nivolumab monotherapy, regardless of LAG-3 expression.[21]

A robust immunohistochemistry (IHC) assay was developed to detect LAG-3 expression by ICs. The assay was used to stratify patients enrolled in RELATIVITY-047, based on the percentage of LAG-3-positive ICs with a morphological resemblance to lymphocytes relative to all nucleated cells within the tumor region (tumor cells [TCs], intratumoral stroma, and peritumoral stroma [the band of stromal elements directly contiguous with the outer tumor margin]) in samples containing $\geq 100$ viable TCs. This assay is also being used in several ongoing clinical trials evaluating relatlimab. This study presents the specificity, sensitivity, analytical precision, and reproducibility of this assay as an aid to determine LAG-3 expression in melanoma patients using a $\geq 1 \%$ IC expression threshold.

\section{MATERIALS AND METHODS}

\section{Principles of the LAG-3 IHC assay}

The LAG-3 IHC assay was developed using a mouse monoclonal antibody clone 17B4 that was made to a synthetic peptide corresponding to the 30-amino acid extra-loop of the first immunoglobulin domain of LAG-3, 
GPPAAAPGHPLAPGPHPAAPSSWGPRPRRY.[22] The assay was performed on formalin-fixed paraffin-embedded (FFPE) tissue sections mounted on glass slides and included pretreatment to remove endogenous melanin that could interfere with interpretation of LAG-3 staining. Following pretreatment, slides were stained and processed using the 17B4 primary antibody on a Leica BOND-III autostainer (Leica Biosystems, Buffalo Grove, IL).

\section{Materials}

\section{Tissue specimens}

FFPE melanoma specimens and control tonsil tissues were obtained from commercial vendors (Boca Biolistics, Pompano Beach, FL; BiolVT, Westbury, NY; and Avaden Biosciences, Seattle, WA). Sections were cut from each tissue block at 4- $\mu \mathrm{m}$ thickness, placed on positively charged slides, and dried for 1 hour at $60^{\circ} \mathrm{C} \pm 2^{\circ} \mathrm{C}$. Excepting sample stability studies, all cut sections were tested within 2 months of sectioning.

\section{Antibodies}

All experiments were performed with monoclonal LAG-3 antibody 17B4 preparations manufactured from hybridoma cultures for Labcorp, except for analysis of clustered regularly interspaced short palindromic repeats (CRISPR)-engineered LAG-3 knockout cell lines, for which a commercially available LAG-3 17B4 antibody was obtained from LSBio (Cat. \# LS-C18692) or as otherwise noted in the text.[22] For precision studies, 3 independent lots of antibody were produced from the 17B4 hybridoma. The working concentration of the LAG-3 17B4 antibody was $2.5 \mu \mathrm{g} / \mathrm{mL}$. The negative control antibody, mouse monoclonal immunoglobulin G1 (IgG1) clone MOPC-21, was obtained 
from Leica Biosystems (Cat. \# PA0996). Further details on the staining and melanin removal procedures are in the supplemental material and supplemental table 1.

\section{Melanin scoring}

To determine the efficacy of the melanin removal step of the protocol, the amount of melanin pigment in the tumor region was scored on a scale of 0 to $4+$. Definitions for melanin pigment scoring expected on melanoma tissue-stained slides and indications for the evaluability of the melanin interpretation in LAG-3 IHC assay scoring are provided in supplemental table 2.

\section{LAG-3 scoring}

An overview of the LAG-3 scoring method is provided in supplemental figure 1.

Evaluation criteria for staining intensity of LAG-3-positive ICs consisted of weak (1+), moderate (2+), and strong (3+) LAG-3-positive staining (supplemental table 3). In addition to cell-surface expression, LAG-3 protein is also retained in intracellular compartments.[23] Thus, LAG-3 IC positivity was quantified in cells that morphologically resembled lymphocytes with punctate (perinuclear and/or Golgi pattern), cytoplasmic, and/or membranous LAG-3 staining of any intensity above background (supplemental figure 2). LAG-3-positive IC content in the tumor region was visually estimated by microscopic examination by the study pathologists, following group alignment using a reference slide set. A hematoxylin and eosin-stained slide for each melanoma sample tested was reviewed by a pathologist to identify the overall tumor region and confirm the presence of $\geq 100$ TCs. Results were reported as the percentage of LAG-3-positive ICs relative to all nucleated cells (ICs [lymphocytes and macrophages], stromal cells, and TCs) within the overall tumor region. The tumor region included TCs, intratumoral 
stroma, and peritumoral stroma (the band of stromal elements directly contiguous with the outer tumor margin). Normal and/or adjacent uninvolved tissues were not included (supplemental figure 3). The scoring scale was (in \%) $0,1,2,3,4,5,10$, and further increments of 10 up to 100 . Samples with LAG-3-positive IC percentage scores of $\geq 1 \%$ were reported as LAG-3-positive.

The methods for the generation of CRISPR-engineered LAG-3 knockout cell lines, peptide inhibition assay, precision study measurements and reproducibility within the same laboratory and across laboratories, and stability experiments are provided in the supplemental material.

\section{RESULTS}

\section{Components of the LAG-3 IHC assay}

Primary antibody concentration and incubation times for assay components were optimized for appropriate positive staining, staining intensity, and overall staining quality of LAG-3 while minimizing nonspecific background staining. Antibody concentrations of $1.25 \mu \mathrm{g} / \mathrm{mL}, 2.5 \mu \mathrm{g} / \mathrm{mL}, 3.0 \mu \mathrm{g} / \mathrm{mL}$, and $3.5 \mu \mathrm{g} / \mathrm{mL}$ were evaluated, and $2.5 \mu \mathrm{g} / \mathrm{mL}$ was determined to be the optimal concentration.

Detection of LAG-3 in tissues using the 17B4 clone antibody

To investigate the ability of the LAG-3 IHC assay to detect LAG-3 IC expression in human FFPE tissue samples, the assay was used to stain LAG-3 in commercially procured human tonsil tissue. We hypothesized that if the LAG-3 IHC assay detected LAG-3 IC expression, then staining would be present in lymphocytes, but not in nonimmune regions, such as the crypt epithelium. Staining of the tonsil tissues using 
the LAG-3 IHC assay revealed membranous/cytoplasmic staining of LAG-3 in lymphocytes in germinal center and interfollicular regions, but no LAG-3 staining in the crypt epithelium (figure 1A). Additionally, no staining was observed in the slide stained with the mouse IgG isotype control.

The LAG-3 IHC assay was developed to include attenuation of melanin staining from FFPE sections prior to IHC and to minimize the impact of melanin pigment on interpretation of the assay. Examples of different levels of melanin pigmentation are shown in supplemental figure 4. The efficacy of melanin removal from tissue samples using the melanin removal procedure is shown in figures $1 \mathrm{~B}$ and $1 \mathrm{C}$. All melanoma tissue samples selected for further investigation had acceptable negative control staining and melanin pigmentation $\leq 1+$. LAG-3 staining was consistent in bleached and unbleached serial sections from the same tissue block (data not shown).

\section{Specificity and sensitivity of the LAG-3 IHC assay}

To investigate the specificity of the LAG-3 IHC assay, the LAG3 gene was disrupted by CRISPR-mediated mutagenesis in COV434 cell lines. In total, 3 pooled cell lines were derived, each with differing levels of LAG3 knockout (out-of-frame indel frequency = 71.02\% in $\mathrm{Cr} 1,62.07 \%$ in $\mathrm{Cr} 2$, and $65.74 \%$ in $\mathrm{Cr} 3$ ) (figure 2A). The LAG-3 expression of these cell lines was compared with parental COV434 cells to investigate the specificity of the LAG-3 IHC assay. LAG-3 staining in parental COV434 cells was markedly higher than each of the 3 LAG3 knockout cell lines, which each had staining consistent with anticipated levels of residual LAG-3 expression based on the frequency of alterations determined by next-generation sequencing (figure 2B). These data 
suggest that the LAG-3 IHC assay is specific for the detection of LAG-3 protein expression.

A peptide competition assay was performed using a synthetic LAG-3 peptide to further investigate the specificity of the LAG-3 IHC assay. The percentage of LAG-3-positive ICs in melanoma tissue was found to decrease from a starting staining level of $40 \%$ to $<1 \%$ following preincubation with increasing molar ratios of a LAG-3 peptide (table 1), indicating that the LAG-3 peptide bound competitively to the 17B4 clone. 
TABLE 1. LAG-3 IHC peptide competition validation results

\begin{tabular}{|c|c|c|}
\hline $\begin{array}{l}\text { Specimen } \\
\text { (peptide:antibody ratio) }\end{array}$ & $\begin{array}{l}\% \text { LAG-3-positive } \\
\text { ICs }\end{array}$ & Staining intensity \\
\hline Melanoma LAG-3 mAb (0:1) & 40 & $2+$ \\
\hline Melanoma LAG-3 peptide $(1: 0)$ & 0 & $\mathrm{~N} / \mathrm{A}$ \\
\hline Melanoma (1:1) & 40 & $2+$ \\
\hline Melanoma (2:1) & 30 & $2+$ \\
\hline Melanoma $(5: 1)$ & $10-20$ & $1-2+$ \\
\hline Melanoma (10:1) & 2 & $1+$ \\
\hline Melanoma (30:1) & $<1$ & $1+$ \\
\hline
\end{tabular}

ICs, immune cells; IHC, immunohistochemistry; LAG-3, lymphocyte-activation gene 3; mAb, monoclonal antibody; N/A, not applicable.

To determine the range of LAG-3 IC expression in melanoma specimens, 100 commercially procured melanoma samples were assessed using the LAG-3 IHC assay. Of these 100 samples, 38 were positive for LAG-3 IC expression and 62 were negative, using $1 \%$ expression as a cutoff value (figure 3 ). The range of IC expression in the positive specimens was $1 \%$ to $40 \%$, with a median of $3 \%$. Of the positive cases, the majority (36) had a LAG-3 IC staining intensity of 2+, 1 sample had a LAG-3 IC staining intensity of $3+$, and 1 sample had a LAG-3 IC staining intensity of $1+$. Taken together, 
these data indicate that the LAG-3 IHC assay detects varying levels of immune infiltrates expressing LAG-3 in human FFPE melanoma samples. Figure 4 shows representative tissue examples of staining from $0 \%$ to $30 \%$.

\section{Analytical precision of the LAG-3 IHC assay within the same laboratory}

Twenty-four FFPE melanoma samples and 1 normal human tonsil tissue control sample were stained on 2 different Leica BOND-III instruments and subsequently scored by 2 independent pathologists to establish the repeatability and reproducibility of the LAG-3 IHC assay. The intrarun repeatability, interday, interinstrument, interoperator, and interreagent lot reproducibility all demonstrated a high concordance, with all point estimates $>95 \%$ in average negative agreement (ANA), average positive agreement (APA), and overall percentage agreement (OPA) (table 2). 
TABLE 2. Summary of precision study results

Evaluation $\quad$ Percentage agreement $(95 \% \mathrm{Cl})$

\begin{tabular}{ll}
\hline & ANA: $98.5(97.3-99.6)$ \\
Intrarun repeatability & APA: $98.6(97.4-99.6)$
\end{tabular}

OPA: 98.5 (97.3-99.6)

ANA: $97.4(96.4-98.4)$

Interday reproducibility $\quad$ APA: 97.6 (96.6-98.5)

OPA: 97.5 (96.5-98.4)

ANA: 97.8 (96.8-98.6)

Interinstrument reproducibility $\quad$ APA: 97.9 (97.0-98.7)

OPA: 97.8 (97.0-98.6)

ANA: $97.8(96.8-98.6)$

Interoperator reproducibility $\quad$ APA: 97.9 (97.0-98.7)

OPA: 97.8 (96.9-98.7)

ANA: $97.4(96.6-98.2)$

Interreagent lot reproducibility $\quad$ APA: $97.6(96.8-98.3)$

OPA: 97.5 (96.7-98.3)

ANA, average negative agreement; APA, average positive agreement; $\mathrm{Cl}$, confidence interval; OPA, overall percentage agreement. 
Interobserver and intraobserver reproducibility of the LAG-3 IHC assay within the same laboratory

Evaluations of 60 melanoma samples performed by 3 independent pathologists from the same laboratory and repeat evaluations of the same 60 melanoma samples by the same pathologist were examined to determine the interobserver and intraobserver reproducibility of the assay within the same laboratory. To determine the interobserver reproducibility of the LAG-3 IHC assay, pairwise comparisons were made of the 180 diagnostic calls by the 3 pathologists: 91 were concordant for positive-to-positive calls, and 77 were concordant for negative-to-negative calls. Disagreements occurred in 12 cases, all of which had LAG-3 scores around the 1\% threshold (LAG-3-positive IC content of $0 \%-1 \%$, resulting in a lower point estimate and lower bound $95 \%$ confidence interval $(\mathrm{Cl})$ for ANA compared with APA and OPA. Point estimates for ANA, APA, and OPA were $>90 \%$ with the lower bound $95 \%$ Cls $>85 \%$ (table 3 ).

To determine intraobserver reproducibility of the LAG-3 IHC assay, the 60 samples assessed in the interobserver reproducibility testing were reassessed by the same pathologists, following a wash-out period. Among the 180 comparisons of diagnostic calls between 2 reads by 3 pathologists, 89 were positive-to-positive concordant, 78 were negative-to-negative concordant, 8 were negative-to-positive discordant, and 5 were positive-to-negative discordant. Additionally, the point estimates and lower bound 95\% Cls were $>90 \%$ and $>85 \%$, respectively, in ANA, APA, and OPA (table 3). 
TABLE 3. Percentage agreement and $95 \%$ Cls for interobserver and intraobserver agreement within the same laboratory

\begin{tabular}{ll}
\hline Evaluation & Percentage agreement $(95 \% \mathrm{CI})$ \\
\hline ANA: $92.8(88.31-96.59)$ \\
APterobserver reproducibility $93.8(89.95-97.06)$ \\
OPA: $93.3(89.44-96.66)$ \\
ANA: $92.31(87.74-96.09)$ \\
Intraobserver reproducibility & APA: $93.19(89.22-96.52)$
\end{tabular}

OPA: $92.78(88.89-96.11)$

ANA, average negative agreement; APA, average positive agreement; $\mathrm{Cl}$, confidence interval; OPA, overall percentage agreement.

Interlaboratory and intralaboratory reproducibility of the LAG-3 IHC assay

Two experiments were performed to assess interlaboratory reproducibility: interobserver and intraobserver reproducibility, and overall interlaboratory and intralaboratory reproducibility. First, to investigate the interobserver and intraobserver reproducibility of the LAG-3 IHC assay between different laboratories, 70 melanoma LAG-3-prestained cases were assessed by 3 pathologists at 3 separate laboratories. Second, to determine overall interlaboratory and intralaboratory reproducibility, unstained slides from 24 melanoma cases that had previously been shown to have a range of LAG-3 
expression were tested at 3 separate laboratories. The interobserver and intraobserver reproducibility and overall interlaboratory and intralaboratory reproducibility demonstrated assay staining and scoring concordance with point estimates for all studies at $>90 \%$ in ANA, APA, and OPA and lower bound $95 \%$ Cls $>85 \%$ (table 4 ). 
TABLE 4. Percentage agreement and $95 \% \mathrm{Cls}$ in the interlaboratory reproducibility study

\begin{tabular}{ll}
\hline Evaluation & Percentage agreement $(95 \% \mathrm{Cl})$ \\
\hline ANA: $92.1(89.6-94.4)$ & APA: $94.2(92.4-95.9)$ \\
& OPA: $93.3(91.3-95.2)$ \\
& ANA: $90.2(88.7-91.7)$ \\
Intraobserver reproducibility & APA: $92.9(91.7-94.0)$ \\
OPA: $91.8(90.5-93.0)$ \\
ANA: $95.1(93.3-96.7)$ \\
APtralaboratory reproducibility & APA $96.0(94.5-97.3)$ \\
OPA: $95.6(94.0-97.1)$ \\
Interlaboratory reproducibility & ANA: $93.2(91.9-94.4)$ \\
&
\end{tabular}

OPA: $93.9(92.7-94.9)$

ANA, average negative agreement; APA, average positive agreement; $\mathrm{Cl}$, confidence interval; OPA, overall percentage agreement. 


\section{Slide stability experiments}

To establish the stability of LAG-3 protein in unstained FFPE tissue sections on glass slides for the LAG-3 IHC assay, the concordance of sectioned tissue samples stained after different storage periods was measured. There was 100\% concordance in scoring (positive or negative) at all time points for slides stored at ambient temperatures or 2$8^{\circ} \mathrm{C}$. The LAG-3-positive IC staining intensity results for the tonsil tissue were $100 \%$ concordant from baseline through month 18 at both $2-8^{\circ} \mathrm{C}$ and ambient temperatures, with a decrease in LAG-3 IC staining intensity from $3+$ to $2+$ at month 24 . Although there was some slight variation (increase or decrease) in the percentage of LAG-3positive ICs for some melanoma samples during the course of testing (eg, a case reported as $2 \%$ at week $2,1 \%$ at week 4 , and $2 \%$ at month 2 ), the LAG-3 score (positive or negative) and LAG-3-positive IC staining intensity $(1+, 2+, 3+)$ results were $100 \%$ concordant for individual samples tested at each time point and each temperature. The small differences observed may be attributable to variations in the density of ICs between tissue sections.

\section{DISCUSSION}

LAG-3 is a key immune checkpoint currently being investigated as an I-O therapy for patients with solid tumors and hematological malignancies.[13, 16, 18, 21, 24-26] The development of a robust LAG-3 IHC assay will enable the analysis of IC LAG-3 status in the tumor microenvironment and the correlation between LAG-3 expression status and response to LAG-3-directed oncology treatments. A robust LAG-3 IHC assay that is suitable for clinical trials and clinical use for melanoma is described in this work. The specificity of the assay was demonstrated using cell lines with LAG3 gene disruptions 
and with a peptide antigen competition assay. LAG-3 scoring was reported as the percentage of LAG-3-positive ICs (which morphologically resembled lymphocytes) relative to all nucleated cells within the overall tumor region. $A \geq 1 \%$ cutoff was used to determine LAG-3 positivity. Analytical precision was demonstrated for intrarun repeatability, interday, interinstrument, interoperator, and interreagent lot reproducibility, with concordance $>95 \%$. Pathologist interobserver and intraobserver reproducibility was $>90 \%$ in terms of ANA, APA, and OPA. LAG-3 was observed to be stable in unstained tissues mounted on glass slides, with concordant staining observed in samples stored at both $2-8^{\circ} \mathrm{C}$ and ambient temperatures for up to 24 months. These data demonstrate that this assay can reproducibly determine the proportion of LAG-3-positive ICs within a sample. Despite challenges associated with the scoring of ICs, the LAG-3 IHC assay demonstrated a high level of interobserver reproducibility both within the same laboratory and between independent laboratories.[27, 28]

A particular issue for the interpretation of IHC assays for melanoma tissues is the presence of melanin pigment. Melanin pigmentation can interfere with IHC interpretation, as it may obscure morphological features and is similar in color to the chromogen 3,3'-diaminobenzidine tetrahydrochloride hydrate (DAB), which is commonly used in IHC assays, including the LAG-3 IHC assay described here. The pretreatment method described in this work removed melanin from samples without compromising the LAG-3 antigen and resulted in no samples that could not be interpreted due to excess melanin pigmentation.

One limitation of the studies presented in this work is that a number of preanalytical factors may impact the performance of the LAG-3 IHC assay, including location of the 
tissue assessed (ie, primary vs. metastatic),[29, 30] sample ischemia time, and fixation methods.[31] Additionally, the design of the cut slide stability studies compared LAG-3 staining and IC expression with baseline (time 0), but did not include comparison with other timepoints.

The assay described in this report was utilized to stratify patients based on LAG-3 expression in RELATIVITY-047 (NCT03470922), a phase 2/3 clinical trial in patients with previously untreated metastatic or unresectable melanoma. The trial compared combined nivolumab (anti-PD-1) and relatlimab (anti-LAG-3) treatment with nivolumab monotherapy, and benefit of combination therapy was observed in comparison with nivolumab monotherapy.[21] While the median PFS estimates were longer for patients with LAG-3 expression $\geq 1 \%$ across both treatment groups, a benefit with the combination therapy over nivolumab was observed regardless of LAG-3 expression. [21]

Both the present report and RELATIVITY-047 determined LAG-3 positivity using a $\geq 1 \%$ cutoff.[21] However, the prevalence of LAG-3 positivity observed in other sample sets or patient populations may vary, meaning cutoff values for clinical utility will have to be determined and validated in clinical studies. For instance, Dillon et al reported a higher prevalence of LAG-3 positivity using a $\geq 1 \%$ cutoff in a different set of commercially procured FFPE melanoma samples than in the melanoma samples used in this report.[32] Dillon et al also reported a higher prevalence of LAG-3 positivity in gastric and gastroesophageal cancer samples than in the melanoma samples used in this report. The LAG-3 assay described in this manuscript is currently being utilized in a number of clinical trials for multiple different tumor types. 
bioRxiv preprint doi: https://doi.org/10.1101/2022.02.25.481964; this version posted February 26, 2022. The copyright holder for this preprint (which was not certified by peer review) is the author/funder. All rights reserved. No reuse allowed without permission.

In summary, a robust IHC assay for the determination of LAG-3 IC status in the tumor microenvironment in solid tumor tissues has been developed. 


\section{Acknowledgments}

The authors thank John Feder and Samantha Yost, both of Bristol Myers Squibb, for generating the CRISPR knock-out cell lines. Medical writing and editorial support were provided by Peter Harrison, PhD, and Matthew Weddig of Spark Medica Inc, funded by Bristol Myers Squibb.

\section{Competing Interests}

BM, LJ, JY, CS, JS, and SA are employees of Labcorp. BM, LJ, JY, SA, and JS have stock in Labcorp. KJ, AS-C, and SS are consultants/independent contractors of Labcorp. LD and JW are employees of and have stock in Bristol Myers Squibb. CH has stock in Bristol Myers Squibb. DL had stock in Bristol Myers Squibb at the time the study was performed.

\section{Funding}

This study was supported by Bristol Myers Squibb.

\section{Authors' Contributions}

LJ, JY, BM, and JS designed the studies. LJ led the laboratory operation and procedures to provide stained slides to pathologists. BM was the lead pathologist for the study. BM, AS-C, SS, and KJ analyzed and interpreted the IHC slides and provided LAG-3 scores. JY provided statistical study design, data analyses, and interpretation. CS performed peptide inhibition assay. SA reviewed the data and provided input on the interpretation of the data. JS, CS, LJ, LD, CH, and JW provided input on data analysis and interpretation. LD co-led LAG-3 IHC diagnostic development with Labcorp. LD, JW, and $\mathrm{CH}$ developed the validation strategy, in partnership with Labcorp, and reviewed 
and approved the experimental design and validation reports. JW and $\mathrm{CH}$ served as pathology subject matter experts for LAG-3 IHC assay development. DL oversaw assay verification and optimization experiments in support of assay transfer to Labcorp and trained Labcorp staff on using the LAG-3 IHC assay. $\mathrm{CH}$ trained pathologists at Labcorp on manual scoring of the LAG-3 IHC assay and developed the LAG-3 IHC scoring algorithm and the assay scoring manual used at Labcorp. All authors contributed to drafting, reviewed, and approved the manuscript.

\section{Data availability statement}

The datasets generated during and/or analyzed during the current study are not publicly available but are available from the corresponding author on reasonable request. 


\section{REFERENCES}

1. Vaddepally RK, Kharel P, Pandey R, et al. Review of indications of FDAapproved immune checkpoint inhibitors per NCCN Guidelines with the level of evidence. Cancers (Basel) 2020;12(3):738.

2. Guo L, Wei R, Lin Y, et al. Clinical and recent patents applications of PD-1/PD-L1 targeting immunotherapy in cancer treatment-current progress, strategy, and future perspective. Front Immunol 2020;11:1508.

3. Larkin J, Chiarion-Sileni V, Gonzalez R, et al. Combined nivolumab and ipilimumab or monotherapy in previously untreated melanoma. N Engl J Med 2015;373(1):23-34.

4. Ascierto PA, Del Vecchio M, Mandalá M, et al. Adjuvant nivolumab versus ipilimumab in resected stage IIIB-C and stage IV melanoma (CheckMate 238): 4year results from a multicentre, double-blind, randomised, controlled, phase 3 trial. Lancet Oncol 2020;21(11):1465-77.

5. Eggermont AMM, Blank CU, Mandala M, et al. Adjuvant pembrolizumab versus placebo in resected stage III melanoma (EORTC 1325-MG/KEYNOTE-054): distant metastasis-free survival results from a double-blind, randomised, controlled, phase 3 trial. Lancet Oncol 2021;22(5):643-54.

6. Hellmann MD, Paz-Ares L, Bernabe Caro R, et al. Nivolumab plus ipilimumab in advanced non-small-cell lung cancer. N Engl J Med 2019;381(21):2020-31.

7. Paz-Ares L, Ciuleanu T-E, Cobo M, et al. First-line nivolumab plus ipilimumab combined with two cycles of chemotherapy in patients with non-small-cell lung 
cancer (CheckMate 9LA): an international, randomised, open-label, phase 3 trial. Lancet Oncol 2021;22(2):198-211.

8. Ferris RL, Blumenschein Jr G, Fayette J, et al. Nivolumab for recurrent squamous-cell carcinoma of the head and neck. $N$ Engl J Med 2016;375(19):1856-67.

9. Burtness B, Harrington KJ, Greil R, et al. Pembrolizumab alone or with chemotherapy versus cetuximab with chemotherapy for recurrent or metastatic squamous cell carcinoma of the head and neck (KEYNOTE-048): a randomised, open-label, phase 3 study. Lancet 2019;394(10212):1915-28.

10. Bellmunt J, de Wit R, Vaughn DJ, et al. Pembrolizumab as second-line therapy for advanced urothelial carcinoma. N Engl J Med 2017;376(11):1015-26.

11. Bajorin DF, Witjes JA, Gschwend J, et al. First results from the phase 3 CheckMate 274 trial of adjuvant nivolumab vs placebo in patients who underwent radical surgery for high-risk muscle-invasive urothelial carcinoma (MIUC). J Clin Oncol 2021;39(suppl 6):Abstract 391.

12. Huang R-Y, Francois A, McGray AR, et al. Compensatory upregulation of PD-1, LAG-3, and CTLA-4 limits the efficacy of single-agent checkpoint blockade in metastatic ovarian cancer. Oncoimmunology 2017;6(1):e1249561.

13. Long L, Zhang X, Chen F, et al. The promising immune checkpoint LAG-3: from tumor microenvironment to cancer immunotherapy. Genes Cancer 2018;9(56):176-89. 
14. Camisaschi C, Casati C, Rini F, et al. LAG-3 expression defines a subset of CD4(+)CD25(high)Foxp3(+) regulatory T cells that are expanded at tumor sites. $J$ Immunol 2010;184(11):6545-51.

15. Grosso JF, Kelleher CC, Harris TJ, et al. LAG-3 regulates CD8+ T cell accumulation and effector function in murine self- and tumor-tolerance systems. J Clin Invest 2007;117(11):3383-92.

16. Woo S-R, Turnis ME, Goldberg MV, et al. Immune inhibitory molecules LAG-3 and PD-1 synergistically regulate T-cell function to promote tumoral immune escape. Cancer Res 2012;72(4):917-27.

17. Keane C, Law SC, Gould C, et al. LAG3: a novel immune checkpoint expressed by multiple lymphocyte subsets in diffuse large B-cell lymphoma. Blood Adv 2020;4(7):1367-77.

18. Workman CJ, Cauley LS, Kim I-J, et al. Lymphocyte activation gene-3 (CD223) regulates the size of the expanding $\mathrm{T}$ cell population following antigen activation in vivo. J Immunol 2004;172(9):5450-55.

19. Wang J, Sanmamed MF, Datar I, et al. Fibrinogen-like protein 1 is a major immune inhibitory ligand of LAG-3. Cell 2019;176(1-2):334-47.e12.

20. Matsuzaki J, Gnjatic S, Mhawech-Fauceglia P, et al. Tumor-infiltrating NY-ESO1-specific CD8+ T cells are negatively regulated by LAG-3 and PD-1 in human ovarian cancer. Proc Natl Acad Sci U S A 2010;107(17):7875-80.

21. Tawbi HA, Schadendorf D, Lipson EJ, et al. Relatlimab and nivolumab versus nivolumab in untreated advanced melanoma. N Engl J Med 2022;386(1):24-34. 
22. Baixeras E, Huard B, Miossec C, et al. Characterization of the lymphocyte activation gene 3-encoded protein. A new ligand for human leukocyte antigen class II antigens. J Exp Med 1992;176(2):327-37.

23. Woo S-R, Li N, Bruno TC, et al. Differential subcellular localization of the regulatory T-cell protein LAG-3 and the coreceptor CD4. Eur J Immunol 2010;40(6):1768-77.

24. ClinicalTrials.gov. A Study to Assess Adjuvant Immunotherapy With Relatlimab and Nivolumab Versus Nivolumab Alone After Complete Resection of Stage III-IV Melanoma (RELATIVITY-098). 2022.

\section{https://clinicaltrials.gov/ct2/show/NCT05002569: Accessed February 11}

25. ClinicalTrials.gov. A Study to Evaluate the Safety, Tolerability, and Efficacy of Relatlimab in Relapsed or Refractory B-Cell Malignancies. 2022.

\section{https://clinicaltrials.gov/ct2/show/NCT02061761: Accessed February 11}

26. Morgensztern D, Chaudhry A, lanotti N, et al. 1359TiP RELATIVITY-104: Firstline relatlimab (RELA) + nivolumab (NIVO) with chemotherapy vs nivo with chemotherapy in stage IV or recurrent non-small cell lung cancer (NSCLC): A phase II, randomized, double-blind study. Annals of Oncology 2021;5:S1030.

27. Adam J, Le Stang N, Rouquette I, et al. Multicenter harmonization study for PDL1 IHC testing in non-small-cell lung cancer. Ann Oncol 2018;29(4):953-58.

28. Rimm DL, Han G, Taube JM, et al. A prospective, multi-institutional, pathologistbased assessment of 4 immunohistochemistry assays for PD-L1 expression in non-small cell lung cancer. JAMA Oncol 2017;3(8):1051-58. 
29. Peng L, Zhang Z, Zhao D, et al. Discordance of immunohistochemical markers between primary and recurrent or metastatic breast cancer: A retrospective analysis of 107 cases. Medicine (Baltimore) 2020;99(25):e20738.

30. Rozenblit M, Huang R, Danziger N, et al. Comparison of PD-L1 protein expression between primary tumors and metastatic lesions in triple negative breast cancers. J Immunother Cancer 2020;8(2):e001558.

31. Ramos-Vara JA, Miller MA. When tissue antigens and antibodies get along: revisiting the technical aspects of immunohistochemistry--the red, brown, and blue technique. Vet Pathol 2014;51(1):42-87.

32. Dillon LM, Wojcik J, Desai K, et al. Abstract 1625: Distribution and prevalence of LAG-3 expression in samples of melanoma and gastric/gastroesophageal junction cancer. Cancer Res 2021;81(Suppl 13):1625-25. 


\section{Figures}

FIGURE 1. Identification of LAG-3 in human tissues using the LAG-3 IHC assay. A, Detection of LAG-3 in human tonsil tissue. Left-hand image depicts LAG-3 staining pattern in tonsil tissue showing moderate to strong plasma membrane/cytoplasmic staining in lymphocytes in germinal centers and interfollicular region. The crypt epithelium is negative. No staining is seen with negative reagent control (right-hand image). B, Staining of FFPE melanoma samples with negative reagent control (upper) or LAG-3 antibody (lower) before (left) and after (right) melanin removal procedure at 10x magnification. C, Examples of LAG-3 staining in FFPE melanoma samples before (upper) and after (lower) the melanin removal procedure at 20× magnification. FFPE, formalin-fixed paraffin-embedded; IHC, immunohistochemistry; LAG-3, lymphocyteactivation gene 3 . 
bioRxiv preprint doi: https://doi.org/10.1101/2022.02.25.481964; this version posted February 26, 2022. The copyright holder for this preprint (which was not certified by peer review) is the author/funder. All rights reserved. No reuse allowed without permission.

A
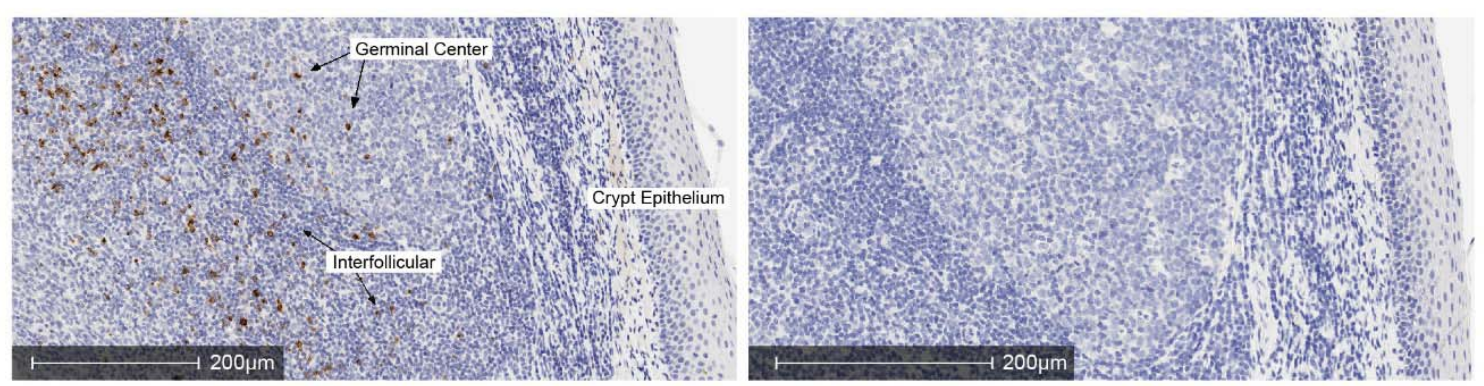

B
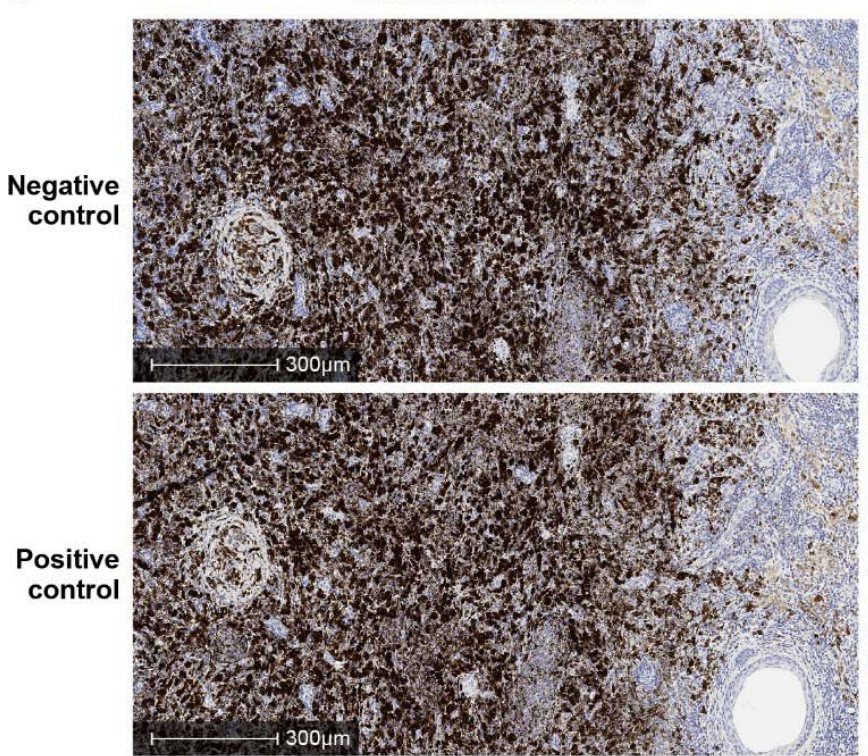

C
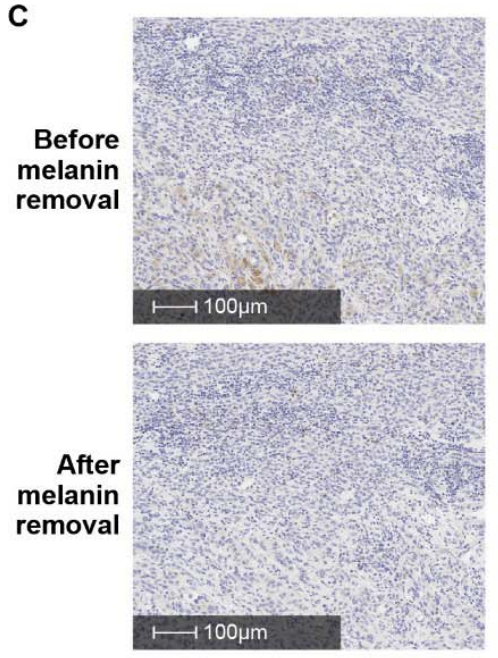
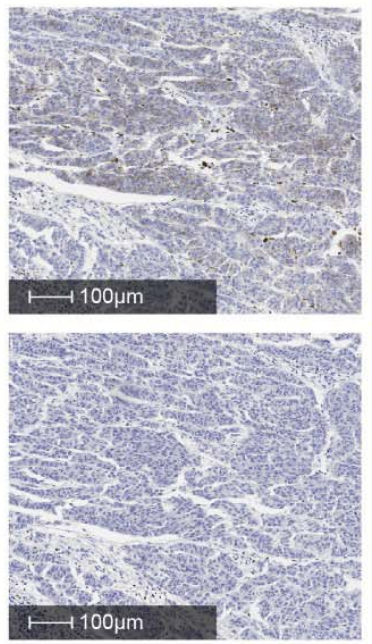
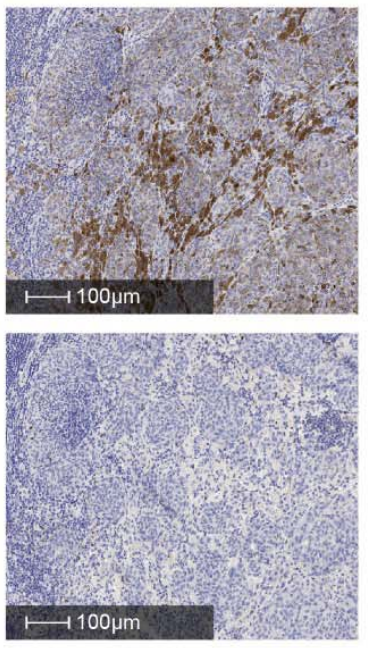

After melanin removal
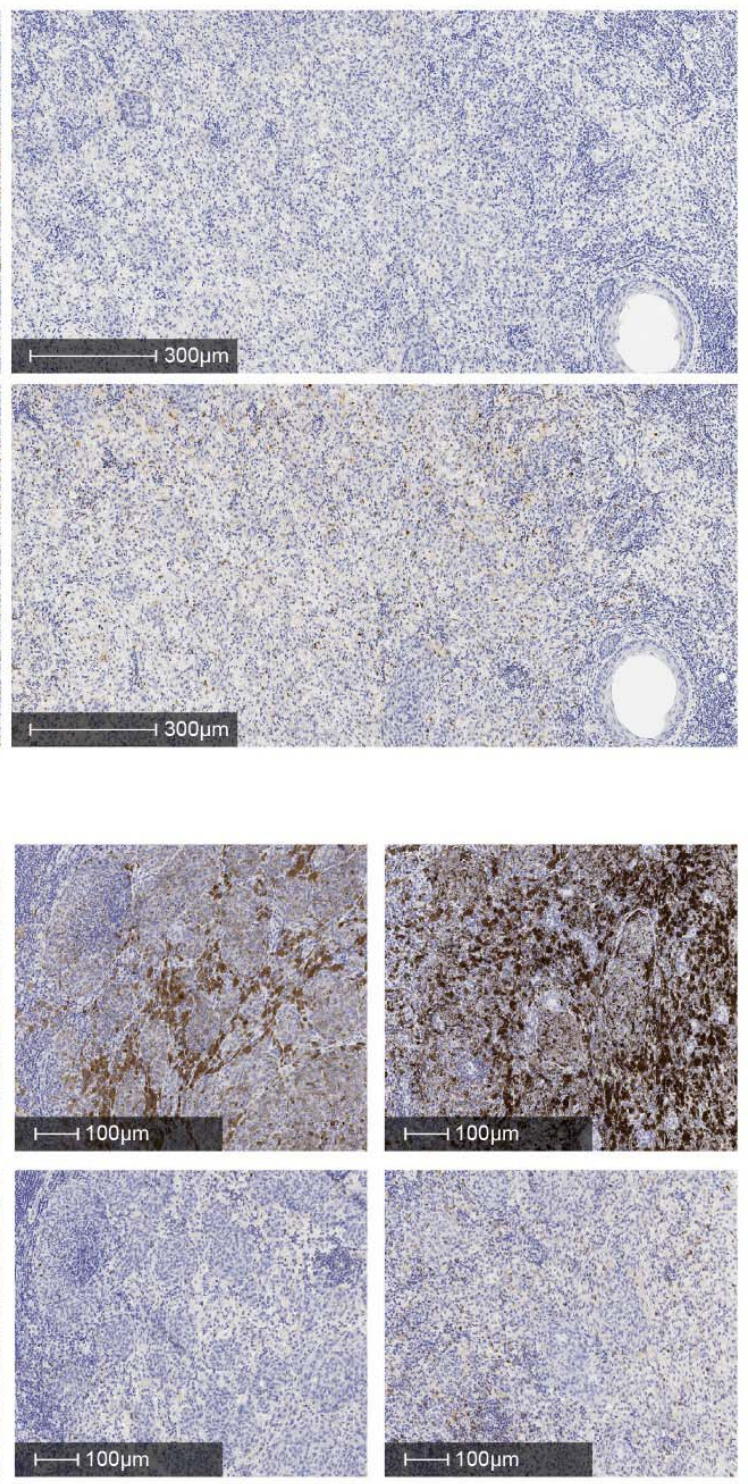
FIGURE 2. Detection of LAG-3 expression in parental COV434 cells and LAG-3disrupted COV434 cells. A, Bar charts showing NGS results from each of the pooled CRISPR-engineered COV434 cell lines. B, IHC staining showing LAG-3 expression in parental COV434 cells and the 3 pooled CRISPR-engineered COV434 cell lines. Tonsil tissue was used as a positive/negative control for the IHC staining. CRISPR, clustered regularly interspaced short palindromic repeats; IHC, immunohistochemistry; LAG-3, lymphocyte-activation gene 3; NGS, next-generation sequencing; WT, wild type.

A

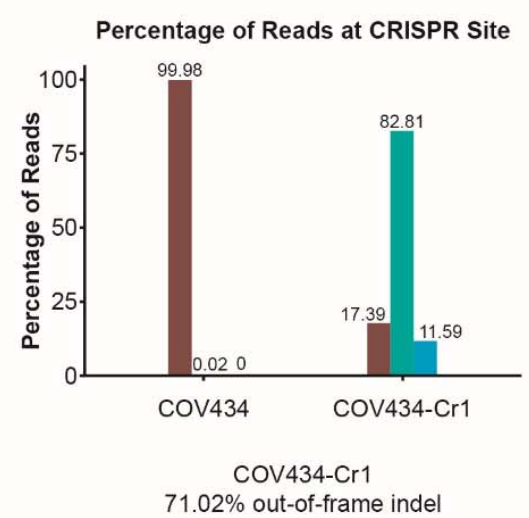

B

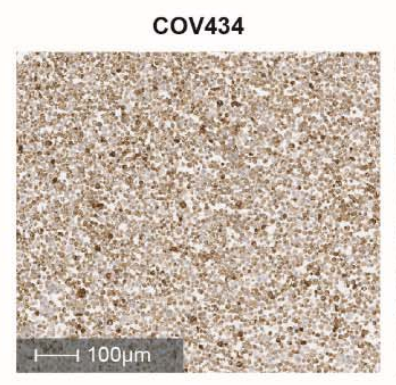

WT $\quad$ W All Indel $\quad$ Inframe Indel

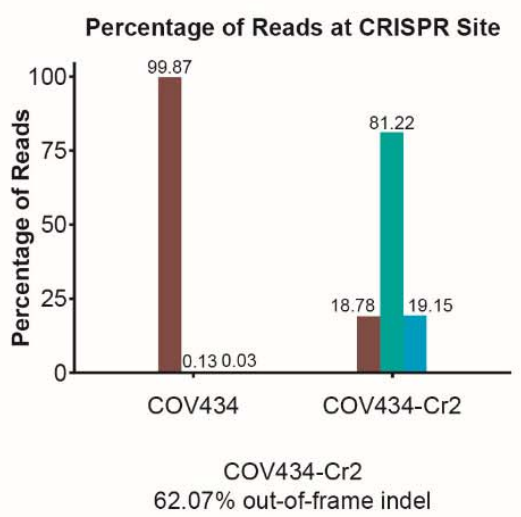

Cov434-Cr1

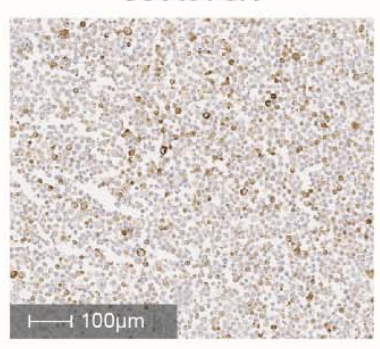

CoV434-Cr2

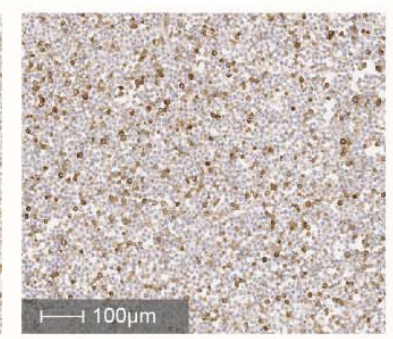

cov434-Cr3

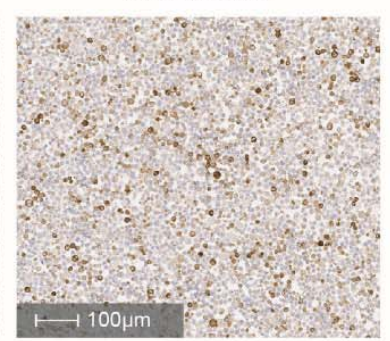


FIGURE 3. Detection of a range of LAG-3 expression levels using the LAG-3 IHC assay. Bar chart showing scoring distribution across LAG-3-positive samples (defined as those with LAG-3-positive IC content $\geq 1 \%$ ) from a set of 100 commercially procured human FFPE melanoma specimens. Of the 100 samples, 38 were LAG-3-positive and 62 were LAG-3-negative. FFPE, formalin-fixed paraffin-embedded; IC, immune cell; IHC, immunohistochemistry; LAG-3, lymphocyte-activation gene 3.

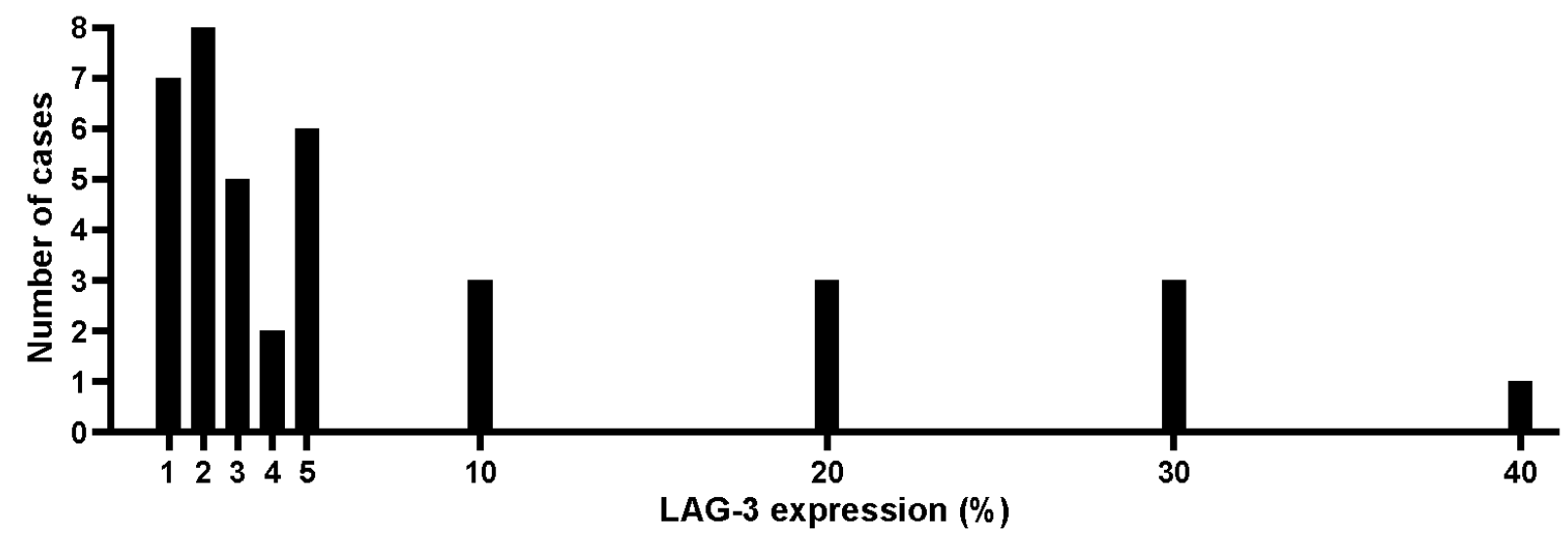


FIGURE 4. Examples of a range of LAG-3 expression levels detected in melanoma tissues using the LAG-3 IHC assay. Melanoma tissues showing a range of staining $(0 \%-30 \%)$ for LAG-3 examined at magnifications of $10 \times$ (left-hand image) and $20 \times$ (right-hand image). IHC, immunohistochemistry; LAG-3, lymphocyte-activation gene 3. 
bioRxiv preprint doi: https://doi.org/10.1101/2022.02.25.481964; this version posted February 26, 2022. The copyright holder for this preprint

(which was not certified by peer review) is the author/funder. All rights reserved. No reuse allowed without permission.

$10 \times$ magnification

$0 \%$

$1 \%$

$2 \%$

$10 \%$

$30 \%$

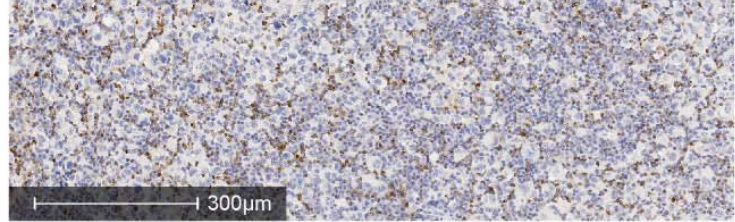

$20 \times$ magnification
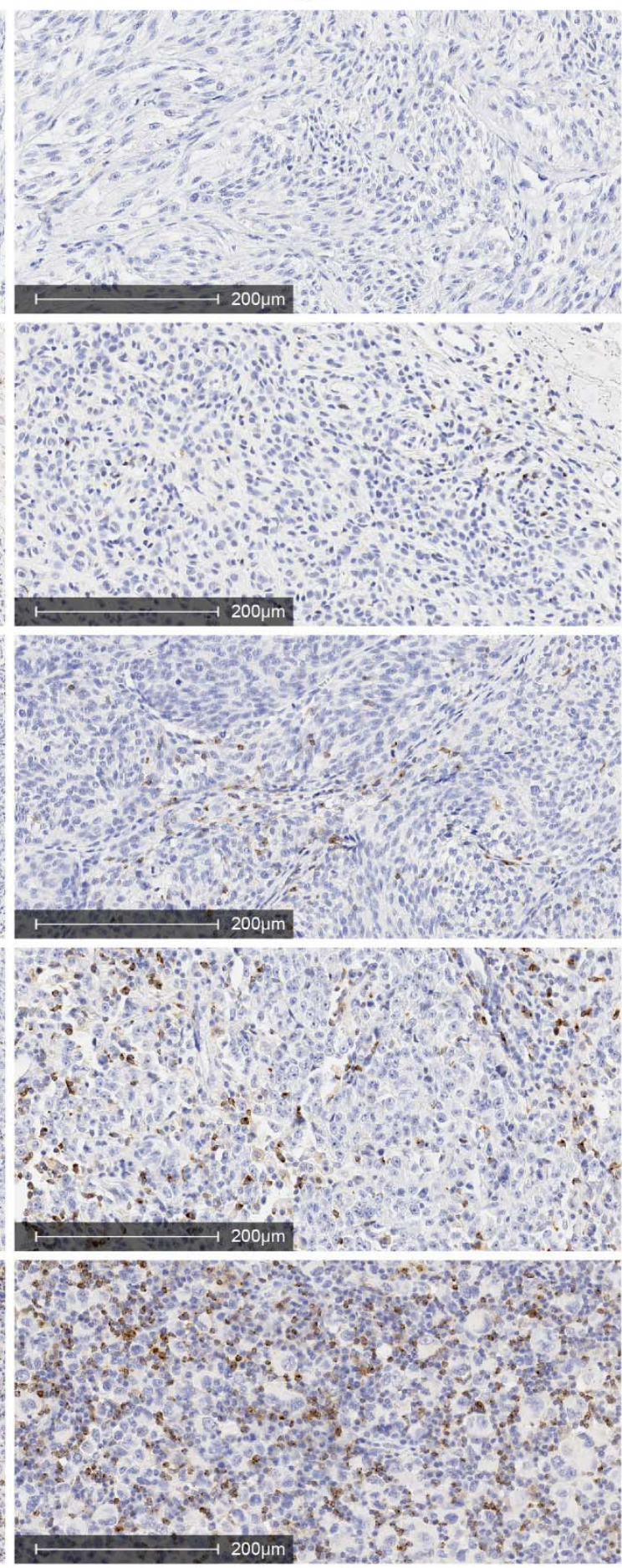